\title{
G3BP1 Depletion Increases Radiosensitisation by Inducing Oxidative Stress in Response to DNA Damage
}

\author{
EUGENE CHO ${ }^{1}$, THOA THI THAN ${ }^{1}$, SU-HYEON KIM ${ }^{1}$, EUN-RAN PARK ${ }^{2}$, \\ MI-YEON KIM ${ }^{2}$, KEE HO LEE ${ }^{2}$ and HYUN JIN SHIN ${ }^{1}$ \\ ${ }^{1}$ Team of Radiation Convergence Research, \\ Korea Institute of Radiological and Medical Sciences, Seoul, Republic of Korea; \\ ${ }^{2}$ Division of Radiation Biomedical Research, \\ Korea Institute of Radiological and Medical Sciences, Seoul, Republic of Korea
}

\begin{abstract}
Background: RAS GTPase-activating proteinbinding protein $(G 3 B P 1)$ is an RNA-binding protein that is essential for assembling stress granules. Many functions related to the survival and progression of cancer have been reported. The current study aimed to investigate the role of G3BP1 in radio-sensitisation of cancer cells. Materials and Methods: Radiation sensitivity and chemosensitivity were analysed in $A 549$ and $H 460$ cells transfected with G3BPI siRNAs, and N-acetyl-L-cysteine (NAC) was used to elucidate the involvement of reactive oxygen species (ROS). Results: G3BP1 depletion sensitised lung cancer cell lines to radiation, and the effect was related to ROS. G3BPI depletion impaired the intracellular ROS scavenging system and NAC abolished the radiation-sensitive phenotypes caused by G3BPI depletion. Conclusion: The study suggested G3BPI as a promising target for radio- and chemosensitisation of lung cancer.
\end{abstract}

Radiation therapy is a central treatment strategy for various types of cancer $(1,2)$. However, treatment efficiency may be compromised due to the radioresistance of cancer cells, and thus the response of patients to radiotherapy may vary considerably. Therefore, increasing sensitivity of cancer cells to radiation, with minimal effect on normal cells, has long been intended in radiotherapy (3-5).

Stress granules (SGs) are cytoplasmic mRNA complexes formed under various stress conditions such as oxidation (sodium arsenite), heat shock, hypoxia, and UV irradiation. They stall initiation of global translation for defence against

Correspondence to: Hyun Jin Shin, Korea Institute of Radiological and Medical Sciences, 215-4 Gongneung-dong, Nowon-Ku, Seoul 01812, Republic of Korea. e-mail: hjshin@kcch.re.kr

Key Words: G3BP1, reactive oxygen species, radio-sensitisation, chemo-sensitization, non-small cell lung cancer. these stress conditions, thereby inhibiting apoptosis (6-8). Several studies have suggested the targeting of SGs as a therapeutic approach in cancer (8-12). SGs have been widely found in cancer tissues, especially in an hypoxic microenvironment, which is the major cause of radioresistance (8-10). In the context of radiosensitisation, SGs might be a valuable target since hypoxia condition in activated SGs are closely related to that in radioresistance (10).

RAS GTPase-activating protein-binding protein (G3BP1) is an RNA-binding protein that is essential in SG formation as an SG-nucleating protein (13-15); it is overexpressed in various tumour tissues, and is related to tumour progression and metastasis $(9,15-18)$. As SGs may have functions related to radiosensitisation as mentioned above, we investigated whether G3BP1, which is a key factor in SG formation, is related to radiosensitisation.

\section{Materials and Methods}

Cell lines. A549 and H460 non-small cell human lung cancer lines were purchased from the American Type Culture Collection (Manassas, VA, USA) and cultured in RPMI medium (Welgene, Gyeongsan, Korea) supplemented with $10 \%$ foetal bovine serum (J $\mathrm{R}$ Scientific, Woodland, CA, USA) and $1 \%(\mathrm{w} / \mathrm{v})$ antibiotics (Welgene) at $37^{\circ} \mathrm{C}$ in a humidified atmosphere containing $5 \% \mathrm{CO}_{2}$.

Small interfering RNAs (siRNAs), plasmids, and transfection. The siRNAs were synthesised, except for the control siRNA that was purchased from Bioneer (Daejeon, Republic of Korea). The sequences used were as follows: $G 3 B P 1$, \#1: 5'-CCAAGAUGAGGUCUUU GGUGGGUUUUU-3'; \#2: 5'-GCGCAUUAACAGUGGUGGGAAA UUAUU-3'. Cells were transfected with siRNA at a final concentration of $20 \mathrm{nM}$, using Lipofectamine RNAiMAX (Invitrogen, Carlsbad, CA, USA) in Opti-MEM (Invitrogen). The knockdown of mRNA by siRNA was confirmed by reverse transcription PCR. cDNA was synthesised using iScript cDNA synthesis kit (Bio-Rad, Carlsbad, CA, USA) and reverse transcription-polymerase chain reaction (RTPCR) was performed using Maxime RT PreMix (Oligo dT) (iNtRON, Seongnam, Republic of Korea). The following primers were used: 
G3BP1: 5'-CCA TTT GAA TCC AAT CCC CCA-3' and 5'-GAG AAG CCT AGT CCC CTG CT-3'; $\beta$-actin: 5'-GGA CTT CGA GCA AGA GAT GG-3' and 5'-AGC ACT GTG TTG GCG TAC AG-3'.

Clonogenic assay. Cells were transfected with siRNA, incubated for $24 \mathrm{~h}$, thereafter seeded at a density of $2 \times 10^{3}$ in $60-\mathrm{mm}$ dishes, incubated again for $24 \mathrm{~h}$, and subsequently irradiated with 2 or 4 Gy using a Gammacell 3000 Elan irradiator $\left({ }^{137} \mathrm{Cs} \gamma\right.$-ray source; MD S Nordin, ON, Canada). Incubation of cells continued until visible colonies were formed. The colonies were then fixed with $10 \%$ formaldehyde and stained with crystal violet. Survival rate was calculated as: number of colonies under irradiated condition/number of colonies under non-irradiated condition. To evaluate chemosensitisation by G3BP1 depletion, clonogenic assay was performed with the same method measuring radiosensitivity. Cells were treated with $40 \mathrm{nM}$ of doxorubicin after low-density seeding for $6 \mathrm{~h}$, then after replacement of the medium, cells were incubated until visible colonies were formed.

Fluorescence microscopy. Cells grown on glass coverslips placed in a 6 well plate were transfected with siRNA, incubated for $24 \mathrm{~h}$, then irradiated with 4 Gy and further incubated for $6 \mathrm{~h}$. For immunostaining, cells were fixed with $10 \%$ formaldehyde in phosphate-buffered saline (PBS) for $10 \mathrm{~min}$ at room temperature, washed with $0.1 \%$ Triton X-100 in PBS (PBS-T), incubated in PBS$\mathrm{T}$ for $10 \mathrm{~min}$, and incubated with blocking solution (3\% skim milk in PBS). The slides were then incubated with primary antibodies against $\gamma-\mathrm{H} 2 \mathrm{~A}$ histone family member X (H2AX) for $2 \mathrm{~h}$ (Abcam, Cambridge, UK). Subsequently, the slides were washed with PBS, incubated with Alexa 488- or 555-conjugated secondary antibodies for $1 \mathrm{~h}$ (Invitrogen), washed again, and mounted using VECTASHIELD Mounting Medium with 4',6-diamidino-2phenylindole (Vector Laboratories, Burlingame, CA, USA). The stained cells were visualised under a fluorescence microscope (Axio Imager M2; Carl Zeiss AB, Stockholm, Sweden).

Immunoblotting. Cells were transfected with siRNA, irradiated after $24 \mathrm{~h}$, further incubated for $24 \mathrm{~h}$, then harvested and lysed with TNN buffer $(120 \mathrm{mM} \mathrm{NaCl}, 40 \mathrm{mM}$ Tris- $\mathrm{HCl}, \mathrm{pH} 8.0,0.5 \% \mathrm{NP}-40)$ supplemented with a protease inhibitor cocktail (GenDEPOT, Barker, TX, USA). The purified proteins were subjected to polyacrylamide gel electrophoresis, transferred to nitrocellulose membranes, and analysed using primary antibodies against G3BP1 (Santa Cruz Biotechnology, Santa Cruz, CA, USA), poly (ADPribose) polymerase (PARP; Cell Signaling Technology, Stockholm, Sweden), $\beta$-actin (Santa Cruz Biotechnology), caspase-3 (Cell Signaling Technology), $\gamma$-H2AX (phospho Ser139; Millipore, Bedford, MA, USA), sulfiredoxin 1 (SRX1; Santa Cruz Biotechnology), glutathione peroxidase 1 (GPX1; Biodesign, Kennebunk, ME, USA), superoxide dismutase 2 (MnSOD, Biodesign), peroxiredoxin 1 and 2 (PRX1, PRX2; Younginfrontier, Seoul, Republic of Korea). After incubation with the appropriate horseradish peroxidase (HRP)-conjugated secondary antibody (Bethyl Laboratories, Montgomery, TX, USA), the proteins were detected by a chemiluminescence detection system (Santa Cruz Biotechnology).

Flow cytometry. Apoptosis, cell cycle distribution, and intracellular reactive oxygen species (ROS) levels were analysed using flow cytometry (FACSCalibur; BD Biosciences, San Jose, CA, USA).
Cells were transfected with siRNA, irradiated with 4 Gy after $24 \mathrm{~h}$, further incubated for $24 \mathrm{~h}$ before eventually prepared for flow cytometric analysis (19). For experiments including ROS scavenger treatment, cells were treated with $5 \mathrm{mM} \mathrm{N}$-acetyl-L-cysteine (NAC; Sigma-Aldrich, St. Louis, MO, USA) for $2 \mathrm{~h}$ before radiation exposure. For cell-cycle analysis, ethanol-fixed cells were incubated with RNase and subsequently stained with $50 \mathrm{ng} / \mathrm{ml}$ propidium iodide (Sigma-Aldrich). Cell-cycle profile was analysed using the FL-2 channel. For apoptosis assays, harvested cells were stained with fluorescein-5-isothiocyanate (FITC)-conjugated annexin V antibody, as per the manufacturer's protocol (BD Pharmingen, San Diego, CA, USA), and then analysed by flow cytometry using FL1 channel. ROS levels were measured by incubating the cells with 2',7'-dichlorofluorescein diacetate (DCF-DA; $10 \mu \mathrm{M}$; SigmaAldrich) for $30 \mathrm{~min}$. Cells were harvested with trypsin, and analysed in the FL-1 channel using FACSCalibur. For statistical analyses, each experiment was individually performed four times.

Comet assay. Alkaline comet assay was performed as per the manufacturer's protocol (Trevigen, MD, USA). Cells were transfected with siRNA for $24 \mathrm{~h}$, exposed to radiation with $4 \mathrm{~Gy}$ and harvested with trypsin immediately or after 1,3 , and $6 \mathrm{~h}$, then embedded $\left(5 \times 10^{3}\right.$ cells $)$ in low-melting agarose and subsequently dropped onto slides. Samples were lysed, rinsed, and treated with alkali to unwind DNA, followed by electrophoresis with the electrophoresis starter kit (Trevigen). SYBR green-stained samples were analysed with a fluorescence microscope (Axio imager M2; Carl Zeiss AB). The tail moment was analysed with comet analysis software (Trevigen) from over 200 cells in each sample. Abnormal tail moments, with upper and lower $10 \%$ in each sample, were eliminated and the mean calculated. For statistical analyses, each experiment was individually performed thrice.

Statistical analyses. All data were analysed as the mean \pm SD. Statistical differences between two means were assessed using Student's $t$-test (unpaired, two-tailed). A value of $p \leq 0.05$ was considered to be statistically significant.

\section{Results}

Silencing of G3BP1 reduced clonogenic survival in response to radiation and doxorubicin. To examine the role of G3BP1 in radio-sensitisation, we performed clonogenic survival assay with A549 and H460 cell lines in which G3BP1 was depleted before cell irradiation. As shown in Figure 1A, each siRNA (\#1, \#2) targeted G3BP1, and irradiation synergistically reduced clonal cell survival. Depletion of G3BP1 by siRNAs was confirmed by RT-PCR (Figure 1B). G3BP1 depletion with siRNA \#1 and \#2 reduced the survival rate after irradiation (4 Gy) from $60 \%$ to $10 \%$ in A549 cells and from $25 \%$ to $8 \%$ (siRNA\#1) and 6\% (siRNA\#2) in H460 cells.

Next, we investigated the function of G3BP1 in chemosensitisation. G3BP1 depletion sensitised A549 and H460 cells to doxorubicin (Figure 1C). The survival rate after doxorubicin treatment was reduced by G3BP1 depletion from $70 \%$ to $10 \%$ (siRNA\#1) and $15 \%$ (siRNA\#2) in A549 cells and from $80 \%$ to $30 \%$ (siRNA\#1) and $20 \%$ (siRNA\#2) in $\mathrm{H} 460$ cells. 
A

B
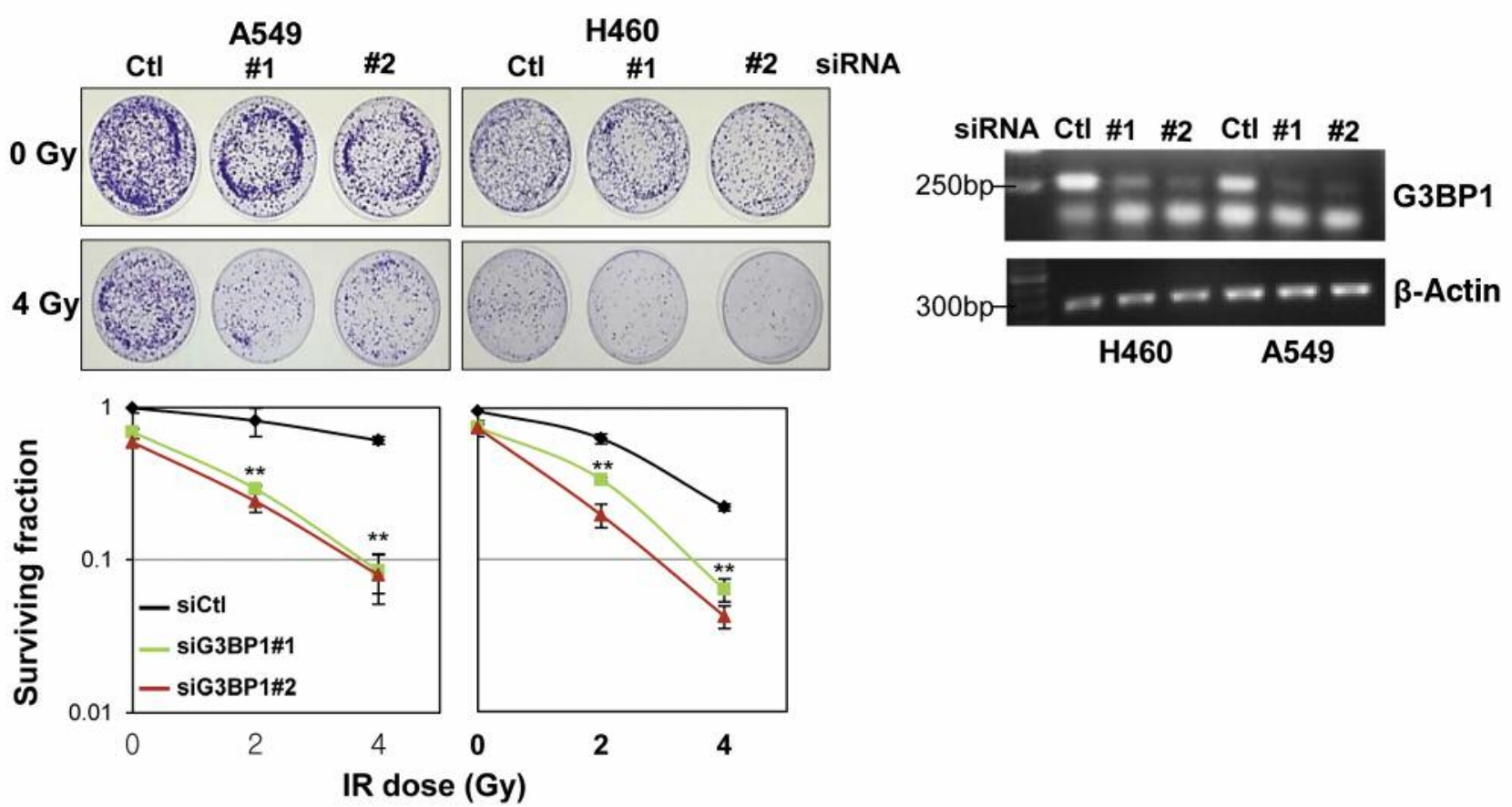

C

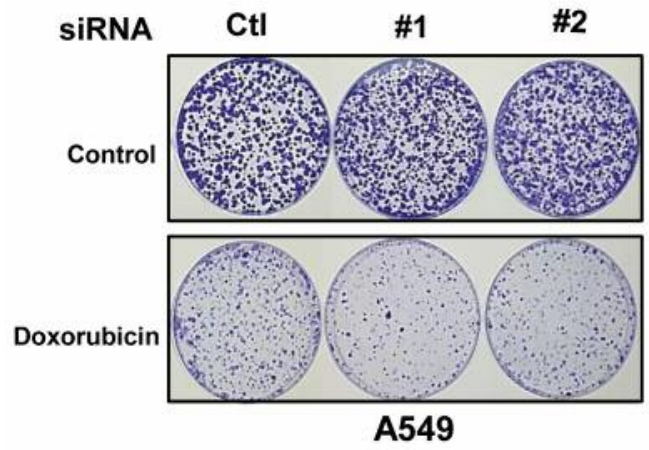

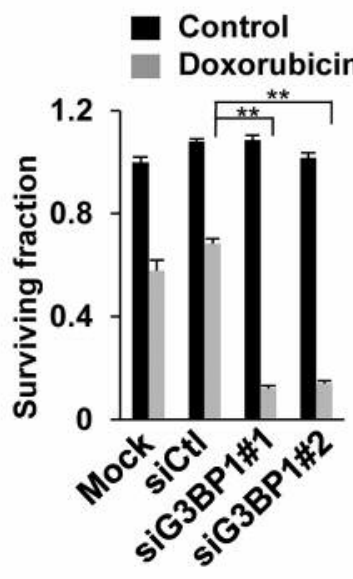

A549

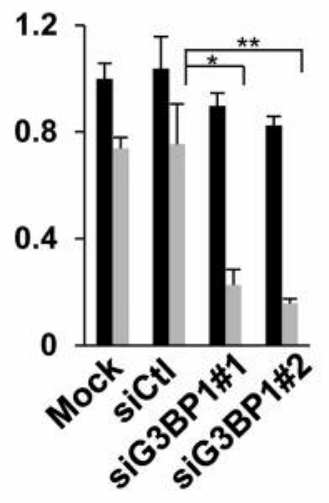

H460

Figure 1. RAS GTPase-activating protein-binding protein (G3BP1) depletion reduces the survival of non-small cell lung cancer cells under irradiation, and doxorubicin treatment. A549 and H460 cell lines were transfected with G3BP1 (\#1 and \#2) or control-siRNA, and then subjected to 4-Gy irradiation or doxorubicin treatment. The surviving fraction was determined by clonogenic assay. Colonies were stained with crystal violet and the survival fraction of each sample was calculated as the ratio of surviving colonies under each condition relative to that under the non-treated condition (A and C). Data are presented as means $\pm S E$ from triplicates. Significantly different at $*_{p}<0.05$ and ${ }^{* *} p<0.01$. The assay was confirmed by three independent experiments having similar results. Depletion of G3BP1 by siRNA was confirmed by reverse transcription-polymerase chain reaction (B). siRNA, small interfering $R N A ;$ ctl, control, IR, irradiation.

Silencing of G3BP1 increased radiation-induced DNA damage and apoptosis. To analyse the cellular phenotypes in radiosensitisation by G3BP1 depletion, we investigated DNA damage and apoptosis in A549 and H460 cells. Cells were irradiated after depletion by siRNAs for $24 \mathrm{~h}$ and all experiments were assayed at $12 \mathrm{~h}$ after irradiation. As shown in Figure 2A, PARP cleavage, $\gamma$-H2AX phosphorylation, and caspase- 3 cleavage were synergistically increased due to the combination of G3BP1 depletion and irradiation in A549 cell line, thereby indicating the DNA damage and apoptosis 
A

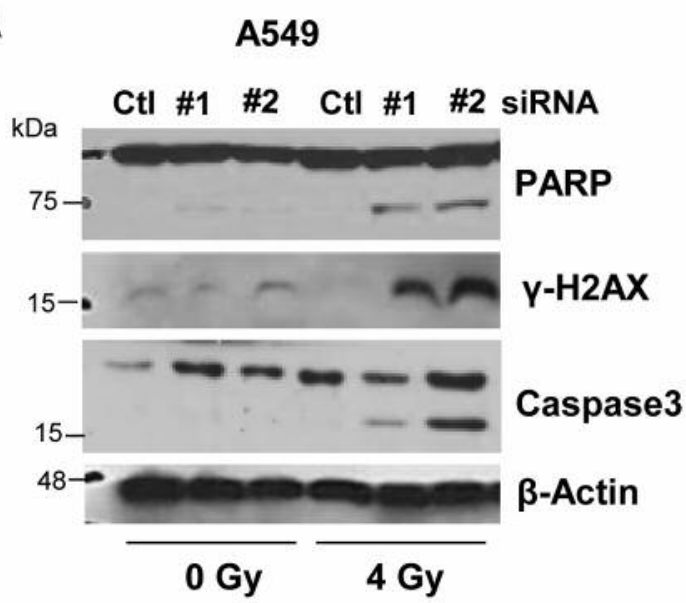

C
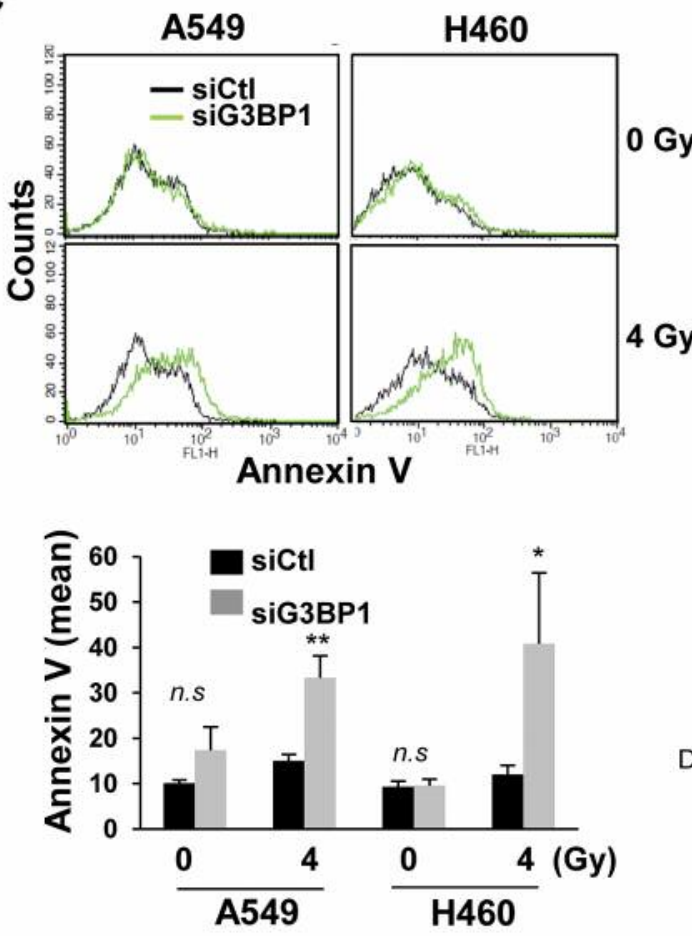

B

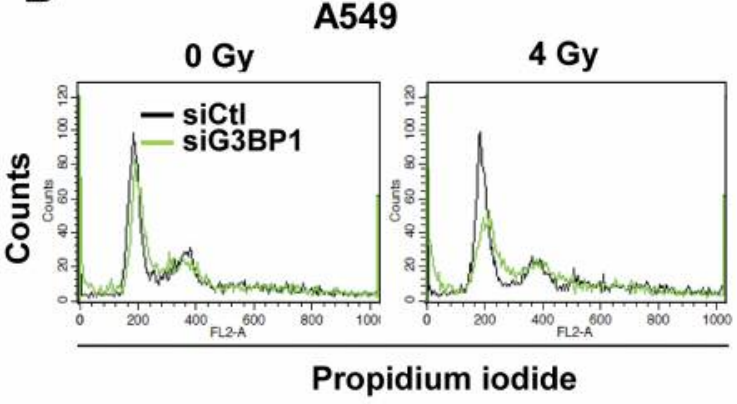

D
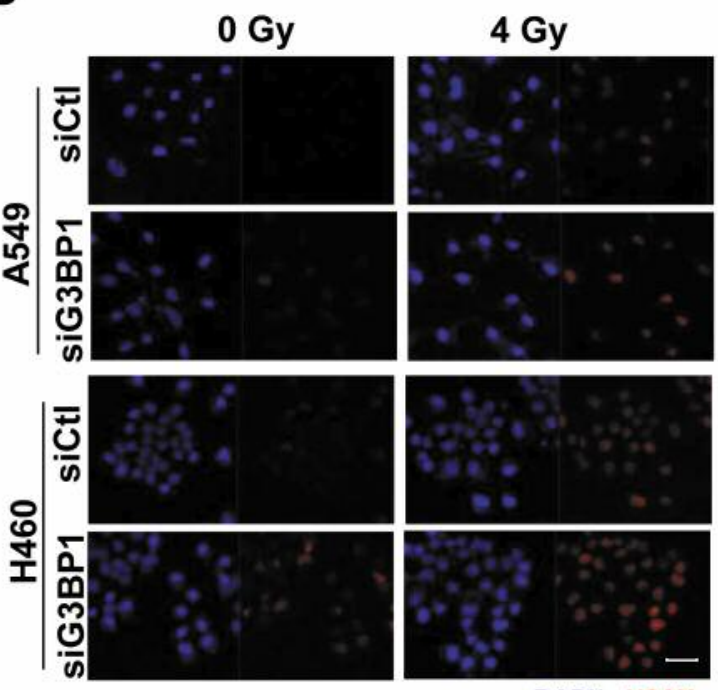

DAPI/Y-H2AX

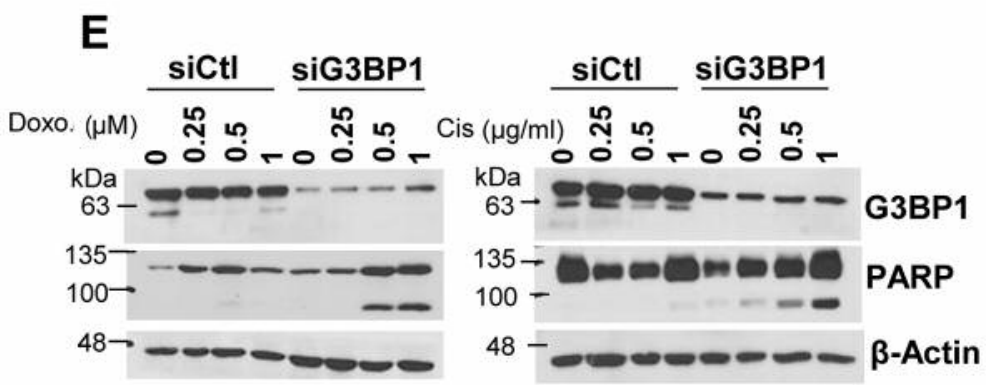

A549

Figure 2. RAS GTPase-activating protein-binding protein (G3BP1) depletion increases apoptosis of non-small cell lung cancer cells after irradiation or anticancer drug treatment. G3BP1-or control siRNA-transfected A549 and H460 cells were irradiated with 4 Gy, incubated for 24 h (A-C, E) or $6 \mathrm{~h}(D)$, and prepared for analysis. (A) A549 cell lysates were subjected to western blotting with antibodies against poly (ADP-ribose) polymerase $(P A R P), \gamma-H 2 A$ histone family member $X(H 2 A X)$, caspase 3 , and $\beta$-actin. (B) Cell-cycle profile of cells was analysed with flow cytometry. (C) Apoptotic (annexin-V-positive) cell profiles were analysed with flow cytometry (upper) and the percentages of annexin-V-positive apoptotic cells were determined by analysis of the histograms (lower). Data represent the mean \pm SEM of three independent experiments. Significantly different at ${ }^{*} p<0.05$ and $* * p<0.01 ;$ n.s.: not significantly different. (D) Representative photographs obtained by confocal microscopy at a magnification of $400 \times$, showing the formation of $\gamma-H 2 A X$ foci. Scale bar: $50 \mu m$. (E) siRNA-transfected A549 cells were treated with anticancer drug for 24 h and western blotting was performed to analyse apoptosis through cleavage of PARP. siRNA, small interfering RNA; ctl, control. 
A

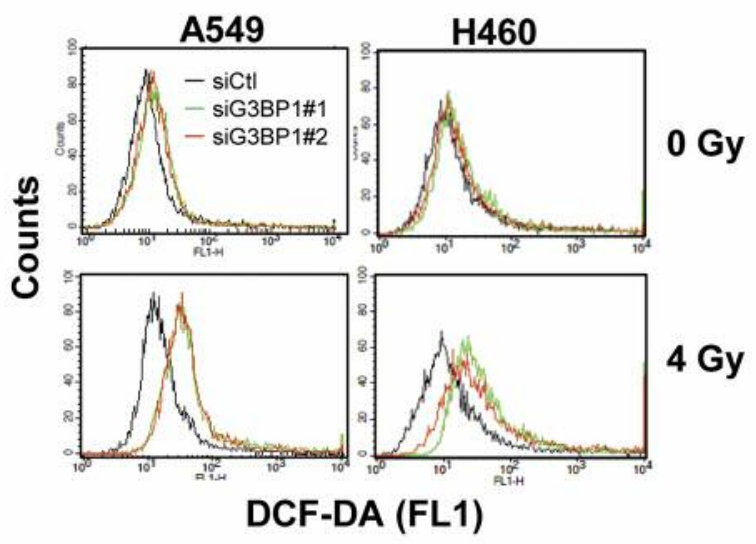

C

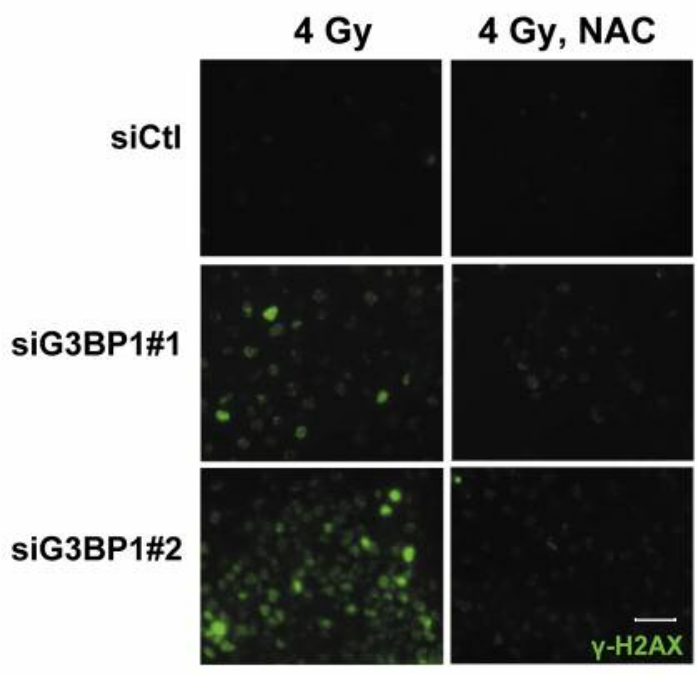

B

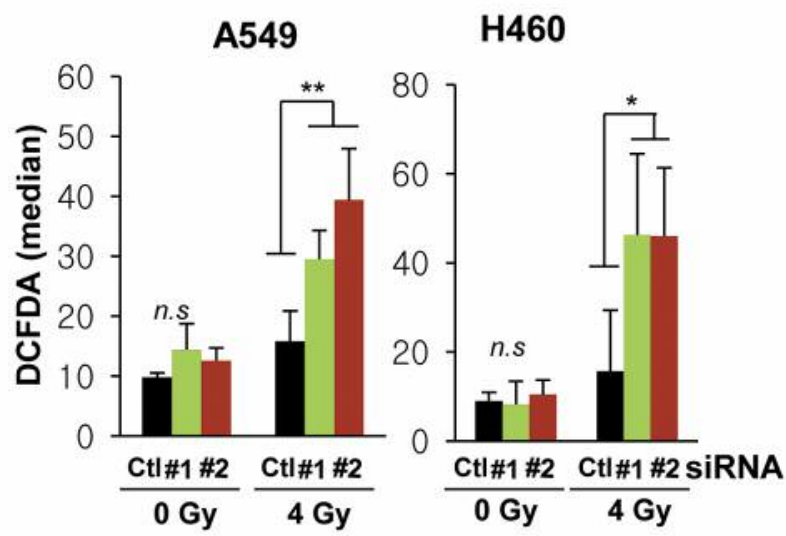

D

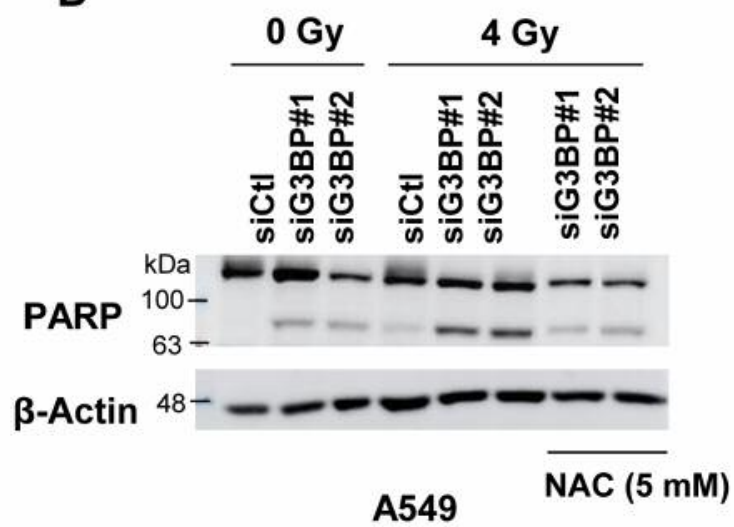

A549

Figure 3. RAS GTPase-activating protein-binding protein (G3BP1) depletion-mediated radiosensitisation depends on oxidative stress. G3BP1- and control siRNA-transfected A549 (A-D) and H460 (A, B) cells were irradiated with $4 \mathrm{~Gy}$, incubated for $6 h(C)$, or $24 h(A, B, D)$, and eventually prepared for analysis. NAC was treated $2 \mathrm{~h}$ before irradiation. A: Levels of intracellular reactive oxygen species (ROS) were determined by measuring fluorescence of 2',7'-dichlorofluorescein diacetate (DCF-DA). The histogram of DCF-DA intensity is a representative of four independent experiments. B: ROS level in each experiment was quantified by median fluorescence. Data represent the mean \pm SEM of four independent experiments. Significantly different at ${ }^{*} p<0.05$ and ${ }^{* *} p<0.01 ;$ n.s.: not significantly different. C: Representative photographs, obtained by fluorescence microscopy at a magnification of $400 \times$, showing the formation of $\gamma-H 2 A$ histone family member X (H2AX) foci in A549 cells. Scale bar: $50 \mu \mathrm{m}$. D: siRNA-transfected A549 cells with/without irradiation under NAC-treated condition were analysed by western blotting for apoptosis. Ctl, Control; siRNA, small interfering RNA.

mediated by radiation to be increased by G3BP1 depletion. Next, we analysed the cell-cycle distribution of A549 cell line under the same condition. The cell cycle pattern was changed, with increased sub G1 population in G3BP1-depleted and irradiated cells (Figure 2B). Apoptosis was quantitatively assessed by measuring annexin $\mathrm{V}$ with flow cytometry (Figure $2 \mathrm{C}$ ). While the increase of apoptosis by G3BP1 depletion was not significantly enhanced in $\mathrm{H} 460$ cells and only slightly enhanced in A549 cells, apoptosis due to irradiation was synergistically increased by G3BP1 depletion in both A549 and $\mathrm{H} 460$ cells. DNA damage in response to irradiation was also increased by G3BP1 depletion, as shown in $\gamma$-H2AX signal in A549 and H460 cells (Figure 2D). We further analysed the effect of G3BP1 on chemosensitisation (using doxorubicin and cisplatin) by western blotting. G3BP1 depletion increased apoptosis after the treatment of A549 cell line with cisplatin and doxorubicin, as shown by the elevated levels of PARP cleavage in Figure 2E. 

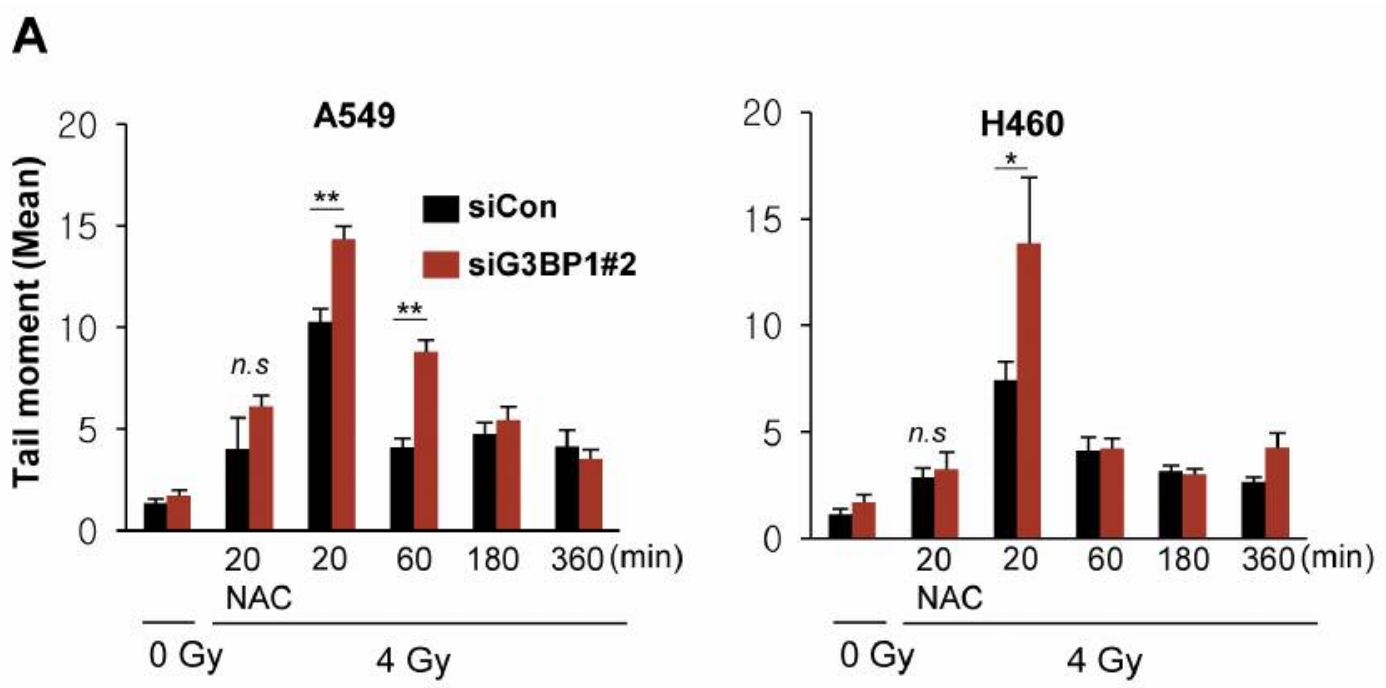

B

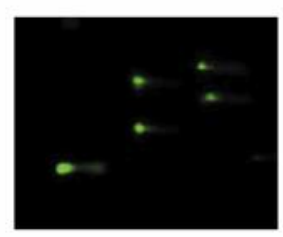

siCon

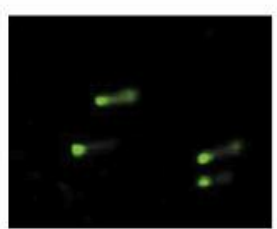

siG3BP1\#2

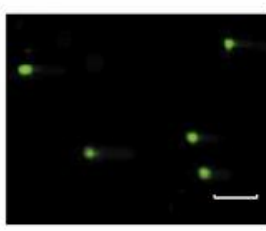

$4 \mathrm{~Gy}, 20 \mathrm{~min}$

A549

C

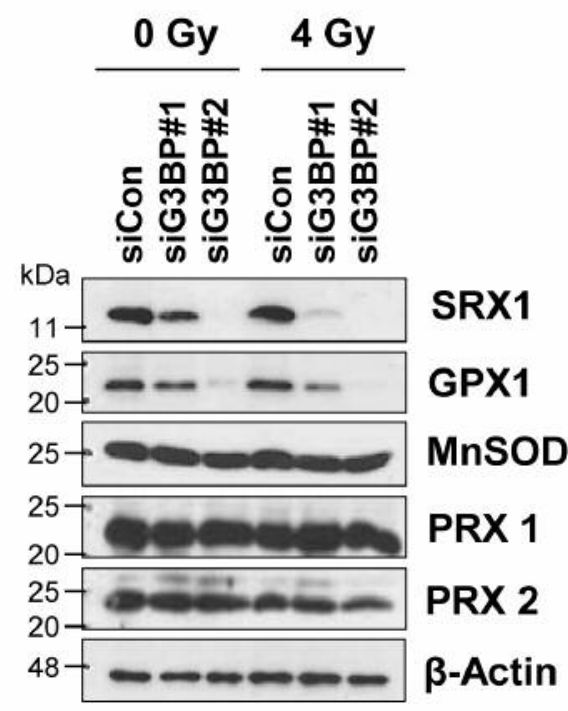

A549

Figure 4. RAS GTPase-activating protein-binding protein (G3BP1) depletion impairs DNA repair in a reactive oxygen species-dependent manner in response to radiation. (A) Comet assay was performed to measure unrepaired DNA damage caused by irradiation. G3BP1 siRNA-transfected cell lines were irradiated for the indicated times. Cells were also treated with $N$-acetyl-L-cysteine (NAC) before irradiation for 2 h. Data represent the mean \pm SEM of the tail moment from three independent experiments. Significantly different at $* p<0.05$ and $* * p<0.01$. (B) Representative photographs of $A 549$ cells were obtained by fluorescence microscopy at a magnification of 400x; scale bar: $50 \mu m$. (C) The expression of main antioxidant enzymes, sulfiredoxin (SRX1), glutathione peroxidase 1 (GPX1), superoxide dismutase 2 (MnSOD), peroxiredoxin 1 and 2 (PRX1, PRX2) was analysed by western blotting in siRNA-transfected and irradiated A549 cells. siRNA, small interfering RNA. 
G3BP1 depletion increased radiosensitivity by regulating $R O S$ production. Since $S G$ s have been reported to play a role in the inhibition of apoptosis by reducing ROS (20), we analysed whether radiosensitisation by G3BP1 depletion might be related to ROS. siRNA-transfected A549 and H460 cells were irradiated and ROS was measured by detecting DCF-DA using flow cytometry (Figure 3A and B). Under non-irradiated condition, depletion of G3BP1 did not significantly affect intracellular ROS levels in either H460 or A549 cells. After irradiation for $3 \mathrm{~h}$, ROS levels increased and became higher than that in G3BP1-depleted cells.

To analyse whether the defect of ROS scavenging upon G3BP1 depletion, under irradiated condition, directly affected cell radiosensitivity, we tested whether pre-treatment with NAC, a ROS scavenging drug, before irradiation would abrogate radiosensitivity induced by G3BP1 depletion. Firstly, DNA damage was analysed by staining siRNAtransfected cells with $\gamma-\mathrm{H} 2 \mathrm{AX}$ in combination with irradiation and NAC treatment. As shown in Figure 3C, under irradiation, G3BP1-depletion induced $\gamma$-H2AX signal, representing DNA damage, which was significantly reduced by NAC treatment in A549 cells. We tested whether NAC affects G3BP1 depletion-mediated radiosensitisation by detecting PARP cleavage with western blotting. G3BP1depletion-induced PARP cleavage in irradiated A549 cell line was reduced by NAC treatment, as shown in Figure 3D.

G3BP1 depletion impaired DNA repair and cellular ROS scavenging under irradiated conditions. To verify whether the function of G3BP1, related to the regulation of ROS and radiosensitisation, affected DNA strand break and repair after radiation damage, we performed a comet assay under the same conditions (Figure 4A and B). Under non-irradiated conditions, the tail moment in control siRNA-transfected cells and G3BP1 siRNA-transfected cells was similar to the basal level. After irradiation, DNA damage was induced promptly and repaired within $1 \mathrm{~h}$. DNA breaks induced by radiation were increased further by G3BP1 depletion. We found the repair process of A549 cells to be delayed, after $1 \mathrm{~h}$ of irradiation. The repair activity after G3BP1 depletion in both cell lines recovered to the same levels as in control cells after $3 \mathrm{~h}$. To determine whether ROS increase due to radiation was related to G3BP1 depletion-mediated increase of tail moment, NAC was applied before irradiation. ROS scavenging upon NAC treatment reduced DNA breaks due to radiation and DNA breaks due to G3BP1 depletion under irradiation were completely blocked by NAC treatment to a level similar to that of control cells.

Next, we tested the expression of representative antioxidant proteins in G3BP1-depleted A549 cells by western blotting (Figure 4C). We found GPX1 and SRX1 proteins to be reduced by G3BP1 depletion in both nonirradiated and irradiated A549 cells, while MnSOD and PRX proteins remained unchanged.

\section{Discussion}

Many types of cancer cells have increased levels of ROS, due to metabolic alteration, mitochondrial defect, or the tumour microenvironment (21), which causes them to be more vulnerable to oxidative insults and more dependent on antioxidant systems compared to normal cells (22). ROS also act as an important factor in radiotherapy, indirectly causing DNA damage $(1,2)$; some cancer types, especially cancer stem cells, have an increased antioxidant mechanism against oxidative stress (23). Therefore, targeting antioxidant mechanisms is a promising cancer-treatment strategy in radiotherapy (23-25). Lung cancer is the most prevalent cause of cancer-related deaths, and non-small cell lung cancer (NSCLC) is the most common type of lung cancer (26). Acquired resistance to radiotherapy or chemotherapy is the most common factor leading to treatment failure in patients with NSCLC. Therefore, studying the sensitisation of targets for radio- and chemotherapy is crucial for reducing their side-effects, and improving clinical outcomes of both in patients with NSCLC.

In the current study, we found that targeting G3BP1 caused radiosensitisation of A549 and H460 NSCLC cells in a ROS-dependent manner. Interestingly, while depletion of G3BP1 had only little cytotoxicity under non-irradiated conditions, it synergistically increased cell death under irradiation in a ROS-dependent manner. We also showed the cytotoxicity of anticancer drugs, namely doxorubicin and cisplatin, to be increased after G3BP1 depletion. These drugs induce ROS which also increases cytotoxicity in cancer cells (27-29); therefore, they may be expected to have a drug combination effect with G3BP1 inhibition.

We planned this study focusing on SGs that function to control free radicals; however, unlike under UV exposure (30), the SG were not assembled under ionising $\gamma$-radiation stress. This implied that G3BP1-depletion-related radiosensitisation may be independent of the function of SGs and some other cellular function may be associated with sensitisation to radiation. G3BP1 is known to play an important part in the regulation of SG dynamics as an RNAbinding protein. However, G3BP1 has also been implicated in cancer progression, invasion, and metastasis, either separately or in relation to $\mathrm{SG}$ formation. It contributes to cancer-related phenotypes by interacting with cancer-related proteins, RNA, or modulating SGs (15). Recently, it was shown to play a role in STING pathway via regulation of cGAS by priming DNA binding and activation (31). This study would also support the benefit of targeting G3BP1 in radiation therapy. Moreover, in cancer tissues, SGs are created due to hypoxic conditions and G3BP1 plays an important role in that; therefore, regulation of radiation sensitisation is expected to be more critical under in vivo conditions than in vitro ones. 
In conclusion, the study has shown G3BP1 to be a candidate target of radio- and chemosensitisation. We found this sensitisation to depend on ROS production by radiation and reduction of antioxidant systems by G3BP1 depletion. Therefore, the deficiency of ROS-scavenging ability induced by G3BP1 depletion does not affect the cell in the normal state, although it increases cytotoxicity by increasing the production of free radicals upon irradiation. Further studies would be required to elucidate the molecular mechanisms regulating the antioxidant system in cancer cells.

\section{Conflicts of Interest}

The Authors declare no competing interest in regard to this study.

\section{Authors' Contributions}

H.J. Shin contributed to the experimental design, performed the experiments, analysed the data and wrote the article. E. Cho. and S. H. Kim performed the experiments and analysed the data. T. T. Than wrote the article and provided resources. E.R. Park, M.Y.Kim, and K.H.Lee. provided resources and analysed the data.

\section{Acknowledgements}

This study was supported by grants from the Korea Institute of Radiological \& Medical Sciences (KIRAMS) funded by the Ministry of Science and ICT (MSIT), Republic of Korea (505312019).

\section{References}

1 Baskar R, Lee KA, Yeo R and Yeoh KW: Cancer and radiation therapy: Current advances and future directions. Int J Med Sci 9(3): 193-199, 2012. PMID: 22408567. DOI: 10.7150/ijms.3635

2 Baskar R, Dai J, Wenlong N, Yeo R and Yeoh KW: Biological response of cancer cells to radiation treatment. Front Mol Biosci 1(24), 2014. PMID: 25988165. DOI: 10.3389/fmolb.2014.00024

3 Barker HE, Paget JTE, Khan AA and Harrington KJ: The tumour microenvironment after radiotherapy: Mechanisms of resistance and recurrence. Nat Rev Cancer 15(7): 409-425, 2015. PMID: 26105538. DOI: $10.1038 / \mathrm{nrc} 3958$

4 Camphausen $\mathrm{K}$ and Tofilon PJ: Combining radiation and molecular targeting in cancer therapy. Cancer Biol Ther 3(3): 247-250, 2004. PMID: 15107611.

5 Begg AC, Stewart FA and Vens C: Strategies to improve radiotherapy with targeted drugs. Nat Rev Cancer 11(4): 239253, 2011. PMID: 21430696. DOI: $10.1038 / \mathrm{nrc} 3007$

6 Protter DSW and Parker R: Principles and properties of stress granules. Trends Cell Biol 26(9): 668-679, 2016. PMID: 27289443. DOI: $10.1016 /$ j.tcb.2016.05.004

7 Harvey R, Dezi V, Pizzinga $M$ and Willis AE: Posttranscriptional control of gene expression following stress: The role of RNA-binding proteins. Biochem Soc Trans 45(4): 10071014, 2017. PMID: 28710288. DOI: 10.1042/BST20160364

8 Anderson P, Kedersha N and Ivanov P: Stress granules, P-bodies and cancer. Biochim Biophys Acta 1849(7): 861-870, 2015. PMID: 25482014. DOI: 10.1016/j.bbagrm.2014.11.009
9 Somasekharan SP, El-Naggar A, Leprivier G, Cheng H, Hajee S, Grunewald TGP, Zhang F, Ng T, Delattre O, Evdokimova V, Wang Y, Gleave $\mathrm{M}$ and Sorensen PH: YB-1 regulates stress granule formation and tumour progression by translationally activating G3BP1. J Cell Biol 208(7): 913-929, 2015. PMID: 25800057. DOI: $10.1083 /$ jcb.201411047

10 Moeller BJ, Cao Y, Li CY and Dewhirst MW: Radiation activates HIF-1 to regulate vascular radiosensitivity in tumours: Role of reoxygenation, free radicals, and stress granules. Cancer Cell 5(5): 429-441, 2004. PMID: 15144951.

11 Grabocka E and Bar-Sagi D: Mutant KRAS enhances tumor cell fitness by upregulating stress granules. Cell 167(7): 1803-1813.e12, 2016. PMID: 27984728. DOI: 10.1016/j.cell.2016.11.035

12 Vilas-Boas F de AS, da Silva AM, de Sousa LP, Lima KM, Vago JP, Bittencourt LFF, Dantas AE, Gomes DA, Vilela MC, Teixeira MM and Barcelos LS: Impairment of stress granule assembly via inhibition of the eIF2alpha phosphorylation sensitizes glioma cells to chemotherapeutic agents. J Neurooncol 127(2): 253-260, 2016. PMID: 26732083. DOI: 10.1007/s11060-015-2043-3

13 Tourrière H, Chebli K, Zekri L, Courselaud B, Blanchard JM, Bertrand E and Tazi J: The RasGAP-associated endoribonuclease G3BP assembles stress granules. J Cell Biol 160(6): 823-831, 2003. PMID: 12642610 . DOI: $10.1083 /$ jcb.200212128

14 Reineke LC, Dougherty JD, Pierre P and Lloyd RE: Large G3BP-induced granules trigger eIF2 $\alpha$ phosphorylation. Mol Biol Cell 23(18): 3499-3510, 2012. PMID: 22833567. DOI: 10.1091/mbc.E12-05-0385

15 Alam U and Kennedy D: Rasputin a decade on and more promiscuous than ever? A review of G3BPs. Biochim Biophys Acta Mol Cell Res 1866(3): 360-370, 2019. PMID: 30595162. DOI: $10.1016 /$ j.bbamcr.2018.09.001

16 Zhang H, Ma Y, Zhang S, Liu H, He H, Li N, Gong Y, Zhao S, Jiang JD and Shao RG: Involvement of Ras GTPase-activating protein $\mathrm{SH} 3$ domain-binding protein 1 in the epithelial-tomesenchymal transition-induced metastasis of breast cancer cells via the SMAD signaling pathway. Oncotarget 6(19): 1703917053, 2015. PMID: 25962958. DOI: 10.18632/oncotarget.3636

17 Min L, Ruan Y, Shen Z, Jia D, Wang X, Zhao J, Sun Y and Gu $\mathrm{J}$ : Overexpression of Ras-GTPase-activating protein $\mathrm{SH} 3$ domain-binding protein 1 correlates with poor prognosis in gastric cancer patients. Histopathology 67(5): 677-688, 2015. PMID: 25809930. DOI: 10.1111/his.12695

18 Dou N, Chen J, Yu S, Gao Y and Li Y: G3BP1 contributes to tumour metastasis via upregulation of SLUG expression in hepatocellular carcinoma. Am J Cancer Res 6(11): 2641-2650, 2016. PMID: 27904777.

19 Kim SH, Park ER, Cho E, Jung WH, Jeon JY, Joo HY, Lee KH and Shin HJ: Mael is essential for cancer cell survival and tumorigenesis through protection of genetic integrity. Oncotarget 8(3): 5026-5037, 2017. PMID: 27926513. DOI: 10.18632/ oncotarget. 13756

20 Takahashi M, Higuchi M, Matsuki H, Yoshita M, Ohsawa T, Oie $\mathrm{M}$ and Fujii M: Stress granules inhibit apoptosis by reducing reactive oxygen species production. Mol Cell Biol 33(4): 815829, 2013. PMID: 23230274. DOI: 10.1128/MCB.00763-12

21 Liou GY and Storz P: Reactive oxygen species in cancer. Free Radic Res 44(5): 479-496, 2010. PMID: 20370557. DOI: 10.3109/10715761003667554

22 Noh J, Kwon B, Han E, Park M, Yang W, Cho W, Yoo W, Khang $\mathrm{G}$ and Lee D: Amplification of oxidative stress by a dual stimuli- 
responsive hybrid drug enhances cancer cell death. Nat Commun 6(6907), 2015. PMID: 25892552. DOI: 10.1038/ncomms 7907

23 Glasauer A and Chandel NS: Targeting antioxidants for cancer therapy. Biochem Pharmacol 92(1): 90-101, 2014. PMID: 25078786. DOI: 10.1016/j.bcp.2014.07.017

24 Diehn M, Cho RW, Lobo NA, Kalisky T, Dorie MJ, Kulp AN, Qian D, Lam JS, Ailles LE, Wong M, Joshua B, Kaplan MJ, Wapnir I, Dirbas FM, Somlo G, Garberoglio C, Paz B, Shen J, Lau SK, Quake SR, Brown JM, Weissman IL and Clarke MF: Association of reactive oxygen species levels and radioresistance in cancer stem cells. Nature 458(7239): 780-783, 2009. PMID: 19194462. DOI: 10.1038 /nature 07733

25 Trachootham D, Zhou Y, Zhang H, Demizu Y, Chen Z, Pelicano H, Chiao PJ, Achanta G, Arlinghaus RB, Liu J and Huang P: Selective killing of oncogenically transformed cells through a ROS-mediated mechanism by $\beta$-phenylethyl isothiocyanate. Cancer Cell 10(3): 241-252, 2006. PMID: 16959615. DOI: 10.1016/j.ccr.2006.08.009

26 Siegel R, Ward E, Brawley O and Jemal A: Cancer stastics, 2011. CA cancer J Clin 61(4): 212-236, 2011. PMID: 21685461. DOI: $10.3322 /$ caac. 20121

27 Pilco-Ferreto $\mathrm{N}$ and Calaf GM: Influence of doxorubicin on apoptosis and oxidative stress in breast cancer cell lines. Int $\mathrm{J}$ Oncol 49(2): 753-762, 2016. PMID: 27278553. DOI: 10.3892/ ijo.2016.3558

28 Marullo R, Werner E, Degtyareva N, Moore B, Altavilla G, Ramalingam SS and Doetsch PW: Cisplatin induces a mitochondrial-ROS response that contributes to cytotoxicity depending on mitochondrial redox status and bioenergetic functions. PLoS One 8(11), e81162, 2013. PMID: 24260552 DOI: $10.1371 /$ journal.pone.0081162
29 Tonino SH, Van Laar J, Van Oers MH, Wang JY, Eldering E and Kater AP: ROS-mediated upregulation of NOXA overcomes chemoresistance in chronic lymphocytic leukemia. Oncogene 30(6): 701-713, 2011. PMID: 20935673. DOI: 10.1038/onc.2010.441

30 Gaillard $\mathrm{H}$ and Aguilera A: A novel class of mRNA-containing cytoplasmic granules are produced in response to UV-irradiation. Mol Biol Cell 19(11): 4980-4992, 2008. PMID: 18768757. DOI: 10.1091/mbc.e08-02-0193

31 Liu ZS, Cai H, Xue W, Wang M, Xia T, Li WJ, Xing JQ, Zhao M, Huang YJ, Chen S, Wu SM, Wang X, Liu X, Pang X, Zhang ZY, Li T, Dai J, Dong F, Xia Q, Li AL, Zhou T, Liu Z gang, Zhang XM and Li T: G3BP1 promotes DNA binding and activation of cGAS. Nat Immunol 20(1): 18-28, 2019. PMID: 30510222. DOI: $10.1038 / \mathrm{s} 41590-018-0262-4$
Received September 30, 2019

Revised October 13, 2019

Accepted October 21, 2019 